\title{
Existence of infinitely many high energy solutions for a class of fractional Schrödinger systems
}

\author{
Qi Li ${ }^{1,2}$, Zengqin Zhao ${ }^{1}$ and Xinsheng $\mathrm{Du}^{1 *}$
}

\author{
"Correspondence: \\ duxinsheng1977@163.com \\ 'School of Mathematical Sciences, \\ Qufu Normal University, Qufu, China \\ Full list of author information is \\ available at the end of the article
}

\begin{abstract}
In this paper, we investigate a class of nonlinear fractional Schrödinger systems

$$
\begin{cases}(-\triangle)^{s} u+V(x) u=F_{u}(x, u, v), & x \in \mathbb{R}^{N} \\ (-\triangle)^{s} v+V(x) v=F_{v}(x, u, v), & x \in \mathbb{R}^{N}\end{cases}
$$

where $s \in(0,1), N>2$. Under relaxed assumptions on $V(x)$ and $F(x, u, v)$, we show the existence of infinitely many high energy solutions to the above fractional Schrödinger systems by a variant fountain theorem.
\end{abstract}

MSC: Primary 35K70; secondary 35B44

Keywords: Fractional Schrödinger system; Variant fountain theorem; Fractional Laplacian

\section{Introduction}

In the work, we are concerned with the existence of infinitely many high energy solutions for the following fractional Schrödinger systems:

$$
\begin{cases}(-\triangle)^{s} u+V(x) u=F_{u}(x, u, v), & x \in \mathbb{R}^{N}, \\ (-\triangle)^{s} v+V(x) v=F_{v}(x, u, v), & x \in \mathbb{R}^{N}\end{cases}
$$

where $s \in(0,1), N>2$ and $F_{u}(x, u, v), F_{v}(x, u, v) \in C\left(\mathbb{R}^{N} \times \mathbb{R} \times \mathbb{R}, \mathbb{R}\right)$. We assume that there exists $F(x, u, v) \in C\left(\mathbb{R}^{N} \times \mathbb{R} \times \mathbb{R}, \mathbb{R}\right)$ such that $\nabla F=\left(F_{u}, F_{v}\right)$, where $\nabla F$ denotes the gradient of $F$ in $(u, v) \in \mathbb{R}^{2}$. The operator $(-\Delta)^{s}$ is the fractional Laplacian of order $s$, which can be defined by the Fourier transform $(-\Delta)^{s} u=\mathcal{F}^{-1}\left(|\xi|^{2 s} \mathcal{F} u\right)$. On the calculation and application of classical fractional differential equations and other aspects in mathematics, we refer the reader to [1-5] and the references therein.

Over the past years, the fractional Laplacian $(-\Delta)^{s}(0<s<1)$, as one of the fundamental nonlocal operators, has increasingly had impact on a number of important fields in science, technology and other fields. As a result, much attention has been focused on the problem of fractional Laplacians. For instance, Teng [6] studied the following fractional

(c) The Author(s) 2020. This article is licensed under a Creative Commons Attribution 4.0 International License, which permits use, sharing, adaptation, distribution and reproduction in any medium or format, as long as you give appropriate credit to the original author(s) and the source, provide a link to the Creative Commons licence, and indicate if changes were made. The images or other third party material in this article are included in the article's Creative Commons licence, unless indicated otherwise in a credit line to the material. If material is not included in the article's Creative Commons licence and your intended use is not permitted by statutory regulation or exceeds the permitted use, you will need to obtain permission directly from the copyright holder. To view a copy of this licence, visit http://creativecommons.org/licenses/by/4.0/ 
Schrödinger equation:

$$
(-\triangle)^{s} u+V(x) u=f(x, u), \quad x \in \mathbb{R}^{N},
$$

and proved the existence of infinitely many nontrivial high or small energy solutions by variant fountain theorems. Du and Mao [7] obtained a sufficient condition for the existence of infinitely many nontrivial high energy solutions by variant fountain theorems for (1.2). Some interesting results can be found in [8-23] and the references therein.

Recently, Di Nezza et al. [16] have proved that $(-\Delta)^{s}$ can be reduced to the standard Laplacian $-\Delta$ as $s \rightarrow 1$. If $s=1$, Eq. (1.2) reduces to the classical Schrödinger equation

$$
-\triangle u+V(x) u=f(x, u), \quad x \in \mathbb{R}^{N} .
$$

With the aid of variational method and critical theorems, for the potential $V(x)$ and nonlinearity $f(x, u)$ under various conditions, the results of existence and multiplicity for Eq. (1.3) have been extensively investigated in the literature; see [24-27] and the references therein.

In recent decades, extensive attention of researchers has been devoted to the existence of solutions to the elliptic systems. Zhang and Zhang [28] considered some nonlinear elliptic systems and obtained the existence of weak solutions by using variational methods. Cao and Tang [29] considered the superlinear elliptic system. They presented the existence of infinitely many solutions which were characterized by the number of nodes of each component under some conditions on the nonlinear term. Pomponio [30] discussed the asymptotically linear cooperative elliptic system at resonance. They proved the existence of a non-zero solution and the existence of $N-1$ pairs of nontrivial solutions due to the difference between the Morse index at zero and the Morse index at infinity by a penalization technique. In recent years, many interesting results have been presented on the class of systems; see [31-38] and the references therein. However, the above literature is concerned with the problem of integer order Laplacian and there is little literature which discusses the Schrödinger systems with fractional order Laplacian. Based on the situation, we consider fractional Schrödinger systems (1.1). In this work, we will show the existence of infinitely many nontrivial high energy solutions by variant fountain theorems.

For convenience, we firstly present the following hypotheses:

$\left(V_{1}\right) \quad V \in C\left(\mathbb{R}^{N}\right)$ satisfies $\inf V(x)>0$ and there exist $r_{0}>0$ and $M>0$ such that $\lim _{|y| \rightarrow \infty}$ meas $\left\{x \in \mathbb{R}^{N}:|x-y| \leq r_{0}, V(x) \leq M\right\}=0$, where meas denotes the Lebesgue measure in $\mathbb{R}^{N}$.

(f $\left.f_{1}\right) F \in C^{1}\left(\mathbb{R}^{N} \times \mathbb{R} \times \mathbb{R}, \mathbb{R}\right), F_{u}(x, u, v) u+F_{v}(x, u, v) v \geq 0$ for all $(x, u, v) \in \mathbb{R}^{N} \times \mathbb{R} \times \mathbb{R}$, $F_{u}(x, u, v) \leq c\left(1+|(u, v)|^{p-1}\right)$ and $F_{v}(x, u, v) \leq c\left(1+|(u, v)|^{q-1}\right)$ for some $2<p, q<2_{s}^{*}$, where $c$ denote different positive constants and $|(u, v)|=\left(u^{2}+v^{2}\right)^{\frac{1}{2}}$.

$\left(f_{2}\right) \lim _{|(u, v)| \rightarrow 0} \frac{F_{u}(x, u, v)}{|(u, v)|}=0$ and $\lim _{|(u, v)| \rightarrow 0} \frac{F_{v}(x, u, v)}{|(u, v)|}=0$ uniformly in $x \in \mathbb{R}^{N}$.

$\left(f_{3}\right)$ There exists $\sigma \in[1, \min \{p, q\})$ such that $\liminf _{|(u, v)| \rightarrow \infty} \frac{F(x, u, v)}{|(u, v)|^{\sigma}} \geq d>0$ uniformly for $x \in \mathbb{R}^{N}$.

$\left(f_{4}\right) \lim _{|(u, v)| \rightarrow \infty} \frac{F(x, u, v)}{|(u, v)|^{2}}=\infty$ uniformly in $x \in \mathbb{R}^{N}$.

$\left(f_{5}\right)$ There exist $\mu>2$ and $c>0$ such that

$$
F_{u}(x, u, v) u+F_{v}(x, u, v) v-\mu F(x, u, v) \geq c\left(1+|(u, v)|^{2}\right), \quad \text { for all } x \in \mathbb{R}^{N}
$$


The paper is arranged as follows. In Sect. 2, we introduce preliminaries for proof of main results and variational setting. In Sect. 3, we present our main results and their proofs.

\section{Preliminaries}

Let us address a Hilbert space

$$
H^{s}\left(\mathbb{R}^{N}\right)=\left\{u \in L^{2}\left(\mathbb{R}^{N}\right): \frac{|u(x)-u(y)|}{|x-y|^{\frac{N}{2}+s}} \in L^{2}\left(\mathbb{R}^{N} \times \mathbb{R}^{N}\right)\right\} .
$$

The space is endowed with the natural norm

$$
\|u\|_{H^{s}}=\left(\int_{\mathbb{R}^{N}}|u(x)|^{2} d x+\int_{\mathbb{R}^{N}} \int_{\mathbb{R}^{N}} \frac{|u(x)-u(y)|^{2}}{|x-y|^{N+2 s}} d x d y\right)^{\frac{1}{2}}
$$

and with the inner product

$$
\langle u, \varphi\rangle=\int_{\mathbb{R}^{N}} u(x) \varphi(x) d x+\int_{\mathbb{R}^{N}} \int_{\mathbb{R}^{N}} \frac{(u(x)-u(y))(\varphi(x)-\varphi(y))}{|x-y|^{N+2 s}} d x d y .
$$

By means of the Fourier transform, the space $H^{s}\left(\mathbb{R}^{N}\right)$ can be defined by

$$
H^{s}\left(\mathbb{R}^{N}\right)=\left\{u \in L^{2}\left(\mathbb{R}^{N}\right): \int_{\mathbb{R}^{N}}\left(1+|\xi|^{2}\right)^{s}|\mathcal{F} u(\xi)|^{2} d \xi<+\infty\right\}
$$

For Eq. (1.2), the Hilbert space $H$ is defined by

$$
H=\left\{u \in H^{s}\left(\mathbb{R}^{N}\right): \int_{\mathbb{R}^{N}}|\xi|^{2 s}|\mathcal{F} u(\xi)|^{2} d \xi+\int_{\mathbb{R}^{N}} V(x)|u|^{2} d x<+\infty\right\},
$$

with the following inner product and norm:

$$
\langle u, \varphi\rangle_{H}=\int_{\mathbb{R}^{N}}|\xi|^{2 s}|\mathcal{F} u(\xi)||\mathcal{F} \varphi(\xi)| d \xi+\int_{\mathbb{R}^{N}} V(x) u \varphi d x
$$

and

$$
\|u\|_{H}=\left(\int_{\mathbb{R}^{N}}|\xi|^{2 s}|\mathcal{F} u(\xi)|^{2} d \xi+\int_{\mathbb{R}^{N}} V(x)|u|^{2} d x\right)^{\frac{1}{2}} .
$$

Then $H \times H$ is a Hilbert space with the following the inner product $\langle\cdot, \cdot\rangle$ and norm for any $(u, v),(\varphi, \psi) \in H \times H$ :

$$
\langle(u, v),(\varphi, \psi)\rangle=\langle u, \varphi\rangle+\langle v, \psi\rangle
$$

and

$$
\|(u, v)\|^{2}=\langle(u, v),(u, v)\rangle=\|u\|_{H}^{2}+\|v\|_{H}^{2} .
$$

Under the hypothesis $\left(V_{1}\right)$, we have the following lemma. 
Lemma 2.1 The Hilbert space $H \times H$ is compactly embedded in $L^{t}\left(\mathbb{R}^{N}\right) \times L^{t}\left(\mathbb{R}^{N}\right)$, where $t \in\left[2,2_{s}^{*}\right)$ and $2_{s}^{*}=\frac{2 N}{N-2 s}$.

Proof Let $\left\{u_{n}, v_{n}\right\} \subset H \times H$ be a sequence such that $u_{n} \rightarrow u, v_{n} \rightarrow v$ in $H$. Then $\left\{u_{n}, v_{n}\right\}$ is bounded in $H \times H$ and $u_{n} \rightarrow u, v_{n} \rightarrow v$ in $L_{\text {loc }}^{t}\left(\mathbb{R}^{N}\right)$ for $t \in\left[2,2_{s}^{*}\right)$. Using the famous Gagliado-Nirenberg inequality, we obtain $u_{n} \rightarrow u, v_{n} \rightarrow v$ in $L^{t}\left(\mathbb{R}^{N}\right)$. Thus, the proof is completed

An element $(u, v) \in H \times H$ is called a weak solution of the systems (1.1), if the equation

$$
\begin{aligned}
& \int_{\mathbb{R}^{N}}|\xi|^{2 s}|\mathcal{F} u(\xi)||\mathcal{F} \varphi(\xi)| d \xi+\int_{\mathbb{R}^{N}} V(x) u \varphi d x+\int_{\mathbb{R}^{N}}|\xi|^{2 s}|\mathcal{F} v(\xi)||\mathcal{F} \psi(\xi)| d \xi \\
& \quad+\int_{\mathbb{R}^{N}} V(x) v \psi d x \\
& \quad=\int_{\mathbb{R}^{N}} F_{u}(x, u, v) \varphi d x+\int_{\mathbb{R}^{N}} F_{v}(x, u, v) \psi d x
\end{aligned}
$$

holds for all $(\varphi, \psi) \in H \times H$. A weak solution of the systems (1.1) corresponds to a critical point of the energy functional

$$
\begin{aligned}
I(u, v)= & \frac{1}{2} \int_{\mathbb{R}^{N}}|\xi|^{2 s}|\mathcal{F} u(\xi)|^{2} d \xi+\frac{1}{2} \int_{\mathbb{R}^{N}} V(x) u^{2} d x \\
& +\frac{1}{2} \int_{\mathbb{R}^{N}}|\xi|^{2 s}|\mathcal{F} v(\xi)|^{2} d \xi+\frac{1}{2} \int_{\mathbb{R}^{N}} V(x) v^{2} d x-\int_{\mathbb{R}^{N}} F(x, u, v) d x
\end{aligned}
$$

that is well defined. Furthermore, $I$ is $C^{1}(H \times H, \mathbb{R})$ functional with derivative given by

$$
\begin{aligned}
\left\langle I^{\prime}(u, v),(\varphi, \psi)\right\rangle= & \int_{\mathbb{R}^{N}}|\xi|^{2 s}|\mathcal{F} u(\xi)||\mathcal{F} \varphi(\xi)| d \xi+\int_{\mathbb{R}^{N}} V(x) u \varphi d x \\
& +\int_{\mathbb{R}^{N}}|\xi|^{2 s}|\mathcal{F} v(\xi)||\mathcal{F} \psi(\xi)| d \xi+\int_{\mathbb{R}^{N}} V(x) v \psi d x \\
& -\int_{\mathbb{R}^{N}} F_{u}(x, u, v) \varphi d x-\int_{\mathbb{R}^{N}} F_{v}(x, u, v) \psi d x .
\end{aligned}
$$

Let $H \times H$ be Banach space with the norm $\|(\cdot, \cdot)\|$ and let $\left\{H_{j}\right\}$ be a sequence of subspace of $H, \operatorname{dim} H_{j}$ is finite for $j \in N$. Set $Y_{k}:=\bigoplus_{j=0}^{k} H_{j}$ and $y_{k}=Y_{k} \times Y_{k}, Z_{k}=\overline{\bigoplus_{j=k+1}^{\infty} H_{j}}$ and $\mathcal{Z}_{k}=Z_{k} \times Z_{k}$, then $H=Y_{k} \oplus Z_{k}$ and $H \times H=\mathcal{Y}_{k} \oplus \mathcal{Z}_{k}$.

Let

$$
B_{k}=\left\{(u, v) \in y_{k}:\|(u, v)\| \leq \rho_{k}\right\}
$$

and

$$
S_{k}=\left\{(u, v) \in \mathscr{Z}_{k}:\|(u, v)\|=r_{k}\right\}
$$

for $\rho_{k}>r_{k}>0$. Consider a classical $C^{1}$-functional $\Phi_{\lambda}(u, v): H \times H \rightarrow \mathbb{R}$ defined by

$$
\Phi_{\lambda}(u, v)=A(u, v)-\lambda B(u, v), \quad \lambda \in[1,2] .
$$

Now, we state two variant fountain theorems which come from the idea of Zou in [39]. 
Theorem 2.2 Assume that the functional $\Phi_{\lambda}(u, v)$ satisfies

$\left(B_{1}\right) \Phi_{\lambda}(u, v)$ maps bounded sets to bounded sets uniformly for $\lambda \in[1,2]$, and $\Phi_{\lambda}(-u,-v)=$ $\Phi_{\lambda}(u, v)$ for all $(\lambda, u, v) \in[1,2] \times H \times H$

$\left(B_{2}\right) B(u, v) \geq 0$ for all $(u, v) \in H \times H$, and $B(u, v) \rightarrow \infty$ as $\|(u, v)\| \rightarrow+\infty$ on any finite dimensional subspace $H \times H$;

$\left(B_{3}\right)$ there exists $\rho_{k}>r_{k}>0$ such that

$$
\begin{aligned}
& a_{k}(\lambda)=\inf _{(u, v) \in \mathcal{Z}_{k},\|(u, v)\|=\rho_{k}} \Phi_{\lambda}(u, v) \geq 0, \\
& b_{k}(\lambda)=\max _{(u, v) \in \mathcal{Y}_{k},\|(u, v)\|=r_{k}} \Phi_{\lambda}(u, v)<0, \quad \forall \lambda \in[1,2],
\end{aligned}
$$

and

$$
d_{k}(\lambda)=\inf _{(u, v) \in \mathcal{Z}_{k},\|(u, v)\| \leq \rho_{k}} \Phi_{\lambda}(u, v) \rightarrow 0 \quad \text { as } k \rightarrow+\infty \text { uniformly for } \lambda \in[1,2] .
$$

Then there exist $\lambda_{n} \rightarrow 1,\left(u_{n}\left(\lambda_{n}\right), v_{n}\left(\lambda_{n}\right)\right) \in y_{n}$ such that

$$
\left.\Phi_{\lambda_{n}}^{\prime}\right|_{y_{n}}\left(u\left(\lambda_{n}\right), v\left(\lambda_{n}\right)\right)=0 \quad \text { and } \quad \Phi_{\lambda_{n}}\left(u\left(\lambda_{n}\right), v\left(\lambda_{n}\right)\right) \rightarrow c_{k}, \quad \text { as } n \rightarrow+\infty
$$

where $c_{k} \in\left[d_{k}(2), d_{k}(1)\right]$. Especially, if $\left\{\left(u\left(\lambda_{n}\right), v\left(\lambda_{n}\right)\right)\right\}$ has a convergent subsequence for every $k$, then $\Phi_{1}$ has infinitely many nontrivial critical points $\left\{u_{k}, v_{k}\right\} \in H \times H \backslash\{0,0\}$ satisfying $\Phi_{1}\left(u_{k}, v_{k}\right) \rightarrow 0^{-}$as $k \rightarrow+\infty$.

Theorem 2.3 Assume that the functional $\Phi_{\lambda}(u, v)$ satisfies

$\left(A_{1}\right) \Phi_{\lambda}(u, v)$ maps bounded sets to bounded sets uniformly for $\lambda \in[1,2]$, and $\Phi_{\lambda}(-u,-v)=$ $\Phi_{\lambda}(u, v)$ for all $(\lambda, u, v) \in[1,2] \times H \times H$;

$\left(A_{2}\right) B(u, v) \geq 0$ for all $(u, v) \in H \times H, A(u, v) \rightarrow+\infty$ or $B(u, v) \rightarrow+\infty$ as $\|(u, v)\| \rightarrow+\infty$; or

$\left(A_{3}\right) B(u, v) \leq 0$ for all $(u, v) \in H \times H, B(u, v) \rightarrow-\infty$ as $\|(u, v)\| \rightarrow+\infty$;

$\left(A_{4}\right)$ there exists $\rho_{k}>r_{k}>0$ such that

$$
b_{k}(\lambda)=\inf _{(u, v) \in \mathcal{Z}_{k},\|(u, v)\|=r_{k}} \Phi_{\lambda}(u, v)>a_{k}(\lambda)=\max _{(u, v) \in \mathcal{Y}_{k},\|(u, v)\|=\rho_{k}} \Phi_{\lambda}(u, v), \quad \forall \lambda \in[1,2] .
$$

Then

$$
b_{k}(\lambda) \leq c_{k}(\lambda)=\inf _{\gamma \in \Gamma_{k}} \max _{(u, v) \in B_{k}} \Phi_{\lambda}(\gamma(u, v)), \quad \forall \lambda \in[1,2]
$$

where $\Gamma_{k}=\left\{\gamma \in C\left(B_{k}, H \times H\right): \gamma\right.$ is odd, $\left.\left.\gamma\right|_{\partial B_{k}}=i d\right\}$ and $k \geq 2$. Furthermore, for almost every $\lambda \in[1,2]$, we have a sequence $\left\{\left(u_{n}^{k}(\lambda), v_{n}^{k}(\lambda)\right)\right\}$ such that

$$
\sup _{n}\left\|\left(u_{n}^{k}(\lambda), v_{n}^{k}(\lambda)\right)\right\|<+\infty, \quad \Phi_{\lambda}^{\prime}\left(u_{n}^{k}(\lambda), v_{n}^{k}(\lambda)\right) \rightarrow 0
$$

and

$$
\Phi_{\lambda}\left(u_{n}^{k}(\lambda), v_{n}^{k}(\lambda)\right) \rightarrow c_{k}(\lambda) \quad \text { as } n \rightarrow+\infty
$$


In order to present our main work by the above variant fountain theorems, we define the functional $A, B$ and $\Phi_{\lambda}(u, v)$ on the space $H \times H$ by

$$
\begin{aligned}
A(u, v)= & \frac{1}{2} \int_{\mathbb{R}^{N}}|\xi|^{2 s}|\mathcal{F} u(\xi)|^{2} d \xi+\frac{1}{2} \int_{\mathbb{R}^{N}} V(x) u^{2} d x+\frac{1}{2} \int_{\mathbb{R}^{N}}|\xi|^{2 s}|\mathcal{F} v(\xi)|^{2} d \xi \\
& +\frac{1}{2} \int_{\mathbb{R}^{N}} V(x) v^{2} d x, \\
B(u, v)= & \int_{\mathbb{R}^{N}} F(x, u, v) d x,
\end{aligned}
$$

and

$$
\Phi_{\lambda}(u, v)=A(u, v)-\lambda B(u, v) .
$$

\section{Proofs of the main results}

In this section, we will present the main results and their proofs.

Lemma 3.1 For any finite dimensional subspace $E$ of $H \times H \backslash\{(0,0)\}$, we claim that there exists a positive constant $\varepsilon_{0}>0$ such that

$$
\operatorname{meas}\left\{x \in \mathbb{R}^{N}:|(u, v)| \geq \varepsilon_{0}\|(u, v)\|\right\} \geq \varepsilon_{0}, \quad \text { for any }(u, v) \in E \text {. }
$$

Proof We argue by contradiction. Assume $\left(u_{n}, v_{n}\right) \in E$ such that

$$
\text { meas }\left\{x \in \mathbb{R}^{N}:\left|\left(u_{n}, v_{n}\right)\right| \geq \frac{1}{n}\left\|\left(u_{n}, v_{n}\right)\right\|\right\}<\frac{1}{n}, \quad \text { for any } n \in N \text {. }
$$

For any $n \in N$, let $\left(\tau_{n}, \omega_{n}\right)=\frac{\left(u_{n}, v_{n}\right)}{\left\|\left(u_{n}, v_{n}\right)\right\|}$, then $\left\|\left(\tau_{n}, \omega_{n}\right)\right\|=1$ and

$$
\text { meas }\left\{x \in \mathbb{R}^{N}:\left|\left(\tau_{n}, \omega_{n}\right)\right| \geq \frac{1}{n}\right\}<\frac{1}{n} .
$$

Using the boundedness of $\left(\tau_{n}, \omega_{n}\right)$, up to a subsequence, assume that $\left(\tau_{n}, \omega_{n}\right) \rightarrow(\tau, \omega)$ with $\|(\tau, \omega)\|=1$ for $(\tau, \omega) \in E$. Since $E$ is a finite dimension space, by Lemma 2.1 and the Hölder inequality, we have

$$
\begin{aligned}
\int_{\mathbb{R}^{N}}\left|\left(\tau_{n}, \omega_{n}\right)-(\tau, \omega)\right|^{2} d x & =\int_{\mathbb{R}^{N}}\left|\left(\tau_{n}-\tau, \omega_{n}-\omega\right)\right|^{2} d x \\
& \leq\left(\int_{\mathbb{R}^{N}}\left|\tau_{n}-\tau\right|^{4} d x\right)^{\frac{1}{2}}\left(\int_{\mathbb{R}^{N}}\left|\omega_{n}-\omega\right|^{4} d x\right)^{\frac{1}{2}} \\
& \rightarrow 0 .
\end{aligned}
$$

On the other hand, because of $(\tau, \omega) \neq(0,0)$, there exists a constant $\rho_{0}>0$ such that

$$
\operatorname{meas}\left\{x \in \mathbb{R}^{N}:|(\tau, \omega)| \geq \rho_{0}\right\} \geq \rho_{0} .
$$


We set

$$
\begin{aligned}
& \Omega_{n}=\left\{x \in \mathbb{R}^{N}:\left|\left(\tau_{n}, \omega_{n}\right)\right|<\frac{1}{n}\right\}, \\
& \Omega_{n}^{c}=\left\{x \in \mathbb{R}^{N}:\left|\left(\tau_{n}, \omega_{n}\right)\right| \geq \frac{1}{n}\right\}, \\
& \Omega_{0}=\left\{x \in \mathbb{R}^{N}:|(\tau, \omega)| \geq \rho_{0}\right\} .
\end{aligned}
$$

From (3.2) and (3.4), there exists $N_{0}$ such that, for $\forall n>N_{0}$, we have

$$
\operatorname{meas}\left(\Omega_{n} \cap \Omega_{0}\right) \geq \operatorname{meas}\left(\Omega_{0}\right)-\operatorname{meas}\left(\Omega_{n}^{c}\right) \geq \frac{\left(N_{0}-1\right) \rho_{0}}{N_{0}} .
$$

Consequently, as $n \rightarrow+\infty$,

$$
\begin{aligned}
\int_{\mathbb{R}^{N}}\left|\left(\tau_{n}, \omega_{n}\right)-(\tau, \omega)\right|^{2} d x & \geq \int_{\Omega_{n} \cap \Omega_{0}}\left|\left(\tau_{n}, \omega_{n}\right)-(\tau, \omega)\right|^{2} d x \\
& \geq \int_{\Omega_{n} \cap \Omega_{0}}\left[\left|\left(\tau_{n}, \omega_{n}\right)\right|^{2}-2\left(\tau_{n}, \omega_{n}\right)(\tau, \omega)+|(\tau, \omega)|^{2}\right] d x \\
& \geq \int_{\Omega_{n} \cap \Omega_{0}}\left[|(\tau, \omega)|^{2}-2\left(\tau_{n}, \omega_{n}\right)(\tau, \omega)\right] d x \\
& \geq \int_{\Omega_{n} \cap \Omega_{0}}\left[|(\tau, \omega)|^{2}-2\left|\left(\tau_{n}, \omega_{n}\right)\right||(\tau, \omega)|\right] d x \\
& \geq \rho_{0}\left(\rho_{0}-\frac{2}{n}\right) \operatorname{meas}\left(\Omega_{n} \cap \Omega_{0}\right) \\
& \geq \rho_{0}\left(\rho_{0}-\frac{2}{n}\right) \frac{\left(N_{0}-1\right) \rho_{0}}{N_{0}} \\
& \geq \frac{\left(N_{0}-1\right) \rho_{0}^{3}}{N_{0}} \\
& >0,
\end{aligned}
$$

which leads to a contradiction. The proof is completed.

Lemma 3.2 Assume that $\left(f_{1}\right)$ and $\left(f_{3}\right)$ hold. Then $B(u, v) \geq 0$ for all $(u, v) \in H \times H$ and $B(u, v) \rightarrow+\infty$ as $\|(u, v)\| \rightarrow+\infty$ on any finite dimensional subspace of $H \times H$.

Proof Obviously, for all $(u, v) \in H \times H, B(u, v) \geq 0$ by the hypothesis $\left(f_{1}\right)$.

Next, for any finite dimensional subspace of $H \times H$, we show that $B(u, v) \rightarrow+\infty$ as $\|(u, v)\| \rightarrow+\infty$. By the hypothesis $\left(f_{3}\right)$, there exists $R>0$ such that

$$
F(x, u, v) \geq d|(u, v)|^{\sigma}, \quad \text { for } x \in \mathbb{R}^{N} \text { and }|(u, v)|>R .
$$

Let $D_{(u, v)}:=\left\{x \in \mathbb{R}^{N}:|(u, v)|>\varepsilon_{0}\|(u, v)\|\right\}$ for $(u, v) \in H \times H \backslash\{(0,0)\}$. by Lemma 3.1, for any $(u, v) \in H \times H$ with $\|(u, v)\| \geq \frac{R}{\varepsilon_{0}}$, we have $|(u, v)|>R$, for all $x \in D_{(u, v)}$. Consequently, for any $(u, v) \in H \times H$ with $\|(u, v)\| \geq \frac{R}{\varepsilon_{0}}$, from $\left(f_{3}\right)$ and (3.6), with the help of Lemma 3.1, 
we get

$$
\begin{aligned}
B(u, v) & =\int_{\mathbb{R}^{N}} F(x, u, v) d x \\
& \geq \int_{D_{(u, v)}} F(x, u, v) d x \\
& \geq \int_{D_{(u, v)}} d|(u, v)|^{\sigma} d x \\
& \geq d \varepsilon_{0}^{\sigma}\|(u, v)\|^{\sigma} \operatorname{meas}\left(D_{(u, v)}\right) \\
& \geq d \varepsilon_{0}^{\sigma+1}\|(u, v)\|^{\sigma} .
\end{aligned}
$$

This implies $B(u, v) \rightarrow+\infty$ as $\|(u, v)\| \rightarrow+\infty$ on any finite dimensional subspace of $H \times H$. The proof is completed.

Lemma 3.3 Assume $\left(f_{1}\right),\left(f_{2}\right)$ and $\left(f_{4}\right)$ hold, then there exist two sequences $\rho_{k}>r_{k}>0$ such that

$$
b_{k}(\lambda)=\inf _{(u, v) \in \mathcal{Z}_{k},\|(u, v)\|=r_{k}} \Phi_{\lambda}(u, v)>a_{k}(\lambda)=\max _{(u, v) \in \mathcal{Y}_{k},\|(u, v)\|=\rho_{k}} \Phi_{\lambda}(u, v), \quad \forall \lambda \in[1,2] .
$$

Proof . For $\forall \varepsilon>0$, by $\left(f_{1}\right)$ and $\left(f_{2}\right)$, there exists $c_{\varepsilon}$ such that

$$
\begin{aligned}
& \left|F_{u}(x, u, v)\right| \leq \varepsilon|(u, v)|+c_{\varepsilon}|(u, v)|^{p-1} \\
& \left|F_{v}(x, u, v)\right| \leq \varepsilon|(u, v)|+c_{\varepsilon}|(u, v)|^{q-1}
\end{aligned}
$$

and

$$
\begin{aligned}
|F(x, u, v)| & =|F(x, u, v)-F(x, 0,0)| \\
& \leq \int_{0}^{1}\left|F_{u}(x, t u, t v)\right||u| d t+\int_{0}^{1}\left|F_{v}(x, t u, t v)\right||v| d t \\
& \leq \varepsilon\left[\frac{1}{2}|(u, v)||u|+\frac{1}{2}|(u, v)||v|\right]+c_{\varepsilon}\left[\frac{1}{p}|(u, v)|^{p-1}|u|+\frac{1}{q}|(u, v)|^{q-1}|v|\right]
\end{aligned}
$$

where $(x, u, v) \in \mathbb{R}^{N} \times H \times H$. Therefore, for $(u, v) \in \mathbb{Z}_{k}$ and $\varepsilon$ small enough, by (3.9) and the Hölder inequality, one has

$$
\begin{aligned}
\Phi_{\lambda}(u, v)= & \frac{1}{2} \int_{\mathbb{R}^{N}}|\xi|^{2 s}|\mathcal{F} u(\xi)|^{2} d \xi+\frac{1}{2} \int_{\mathbb{R}^{N}} V(x) u^{2} d x+\frac{1}{2} \int_{\mathbb{R}^{N}}|\xi|^{2 s}|\mathcal{F} v(\xi)|^{2} d \xi \\
& +\frac{1}{2} \int_{\mathbb{R}^{N}} V(x) v^{2} d x-\lambda \int_{\mathbb{R}^{N}} F(x, u, v) d x \\
\geq & \frac{1}{2}\|(u, v)\|^{2}-\lambda \int_{\mathbb{R}^{N}} \varepsilon\left[\frac{1}{2}|(u, v)||u|+\frac{1}{2}|(u, v)||v|\right] \\
& +c_{\varepsilon}\left[\frac{1}{p}|(u, v)|^{p-1}|u|+\frac{1}{q}|(u, v)|^{q-1}|v|\right] d x \\
\geq & \frac{1}{2}\|(u, v)\|^{2}-\lambda \varepsilon\left(\frac{1}{2}\|(u, v)\|_{2}\|u\|_{2}+\frac{1}{2}\|(u, v)\|_{2}\|v\|_{2}\right)
\end{aligned}
$$




$$
\begin{aligned}
& -\lambda c_{\varepsilon}\left[\frac{1}{p}\|(u, v)\|_{p}^{p-1}\|u\|_{p}+\frac{1}{q}\|(u, v)\|_{q}^{q-1}\|v\|_{q}\right] \\
\geq & \frac{1}{2}\|(u, v)\|^{2}-\lambda \varepsilon\left[\frac{1}{2}\|(u, v)\|_{2}^{2}+\frac{1}{2}\|(u, v)\|_{2}^{2}\right] \\
& -\lambda c_{\varepsilon}\left[\frac{1}{p}\|(u, v)\|_{p}^{p}+\frac{1}{q}\|(u, v)\|_{q}^{q}\right],
\end{aligned}
$$

where $\|\cdot\|_{t}$ denotes the usual norm of $L^{t}\left(\mathbb{R}^{N}\right)$. Let $\beta_{k}(2):=\sup _{(u, v) \in \mathcal{Z}_{k},\|(u, v)\|=1}\|(u, v)\|_{2}$, $\beta_{k}(p):=\sup _{(u, v) \in \mathcal{Z}_{k},\|(u, v)\|=1}\|(u, v)\|_{p}, \beta_{k}(q):=\sup _{(u, v) \in \mathcal{Z}_{k},\|(u, v)\|=1}\|(u, v)\|_{q}$, then $\beta_{k}(2) \rightarrow 0$, $\beta_{k}(p) \rightarrow 0, \beta_{k}(q) \rightarrow 0$ as $k \rightarrow \infty$ (cf.[40]). Consequently,

$$
\begin{aligned}
\Phi_{\lambda}(u, v) \geq & \frac{1}{2}\|(u, v)\|^{2}-\lambda \varepsilon \beta_{k}^{2}(2)\|(u, v)\|^{2}-\frac{1}{p} \lambda c_{\varepsilon} \beta_{k}^{p}(p)\|(u, v)\|^{p}-\frac{1}{q} \lambda c_{\varepsilon} \beta_{k}^{q}(q)\|(u, v)\|^{q} \\
\geq & \left(\frac{1}{2}-\lambda \varepsilon \beta_{k}^{2}(2)\right)\|(u, v)\|^{2}-\frac{1}{p} \lambda c_{\varepsilon} \beta_{k}^{p}(p)\|(u, v)\|^{p} \\
& -\frac{1}{q} \lambda c_{\varepsilon} \beta_{k}^{q}(q)\|(u, v)\|^{q}
\end{aligned}
$$

for all $(u, v) \in \mathcal{Z}_{k}$. We choose the appropriate $\varepsilon>0$ and $\lambda$ such that $\frac{1}{2}-\lambda \varepsilon \beta_{k}^{2}(2) \geq \frac{1}{4}$, and we have

$$
\Phi_{\lambda}(u, v) \geq \frac{1}{4}\|(u, v)\|^{2}-\frac{1}{p} \lambda c_{\varepsilon} \beta_{k}^{p}(p)\|(u, v)\|^{p}-\frac{1}{q} \lambda c_{\varepsilon} \beta_{k}^{q}(q)\|(u, v)\|^{q} .
$$

Note that $p, q>2$; without loss of generality, assume $p<q$, then, for $\|(u, v)\|:=r_{k}:=$ $\left(\frac{8}{p} \lambda c_{\varepsilon} \beta_{k}^{p}(p)+\frac{8}{q} \lambda c_{\varepsilon} \beta_{k}^{q}(q)\right)^{\frac{1}{2-p}}$ or $\|(u, v)\|:=r_{k}:=\left(\frac{8}{p} \lambda c_{\varepsilon} \beta_{k}^{p}(p)+\frac{8}{q} \lambda c_{\varepsilon} \beta_{k}^{q}(q)\right)^{\frac{1}{2-q}}$ for any $(u, v) \in$ $\mathcal{Z}_{k}$, one has

$$
\Phi_{\lambda}(u, v) \geq \frac{1}{8} r_{k}^{2}>0
$$

The above inequality implies that

$$
b_{k}(\lambda)=\inf _{(u, v) \in \mathcal{Z}_{k},\|(u, v)\|=r_{k}} \Phi_{\lambda}(u, v)>0
$$

Therefore, by Lemma 3.1, for any $k \in N$, there is a constant $\varepsilon_{k}>0$ such that

$$
\operatorname{meas}\left(D_{(u, v)}\right) \geq \varepsilon_{k}, \quad \text { for all }(u, v) \in Y_{k} \times Y_{k} \backslash\{(0,0)\}
$$

where $D_{(u, v)}=\left\{x \in \mathbb{R}^{N}:|(u, v)| \geq \varepsilon_{k}\|(u, v)\|\right\}$. By the hypothesis $\left(f_{4}\right)$, for $\forall k \in N$, there is a constant $R_{k}>0$ such that

$$
F(x, u, v) \geq \frac{1}{\varepsilon_{k}^{3}}|(u, v)|^{2}, \quad \text { for all }|(u, v)| \geq R_{k} .
$$

By (3.12), we know that, for $(u, v) \in Y_{k} \times Y_{k} \backslash\{(0,0)\}$ with $\|(u, v)\| \geq \frac{R_{k}}{\varepsilon_{k}}$, we obtain $|(u, v)| \geq$ $R_{k}$ for $x \in D_{(u, v)}$. Therefore, by (3.12) and (3.13) for $(u, v) \in Y_{k} \times Y_{k} \backslash\{(0,0)\}$ with $\|(u, v)\| \geq$ 
$\frac{R_{k}}{\varepsilon_{k}}$, one has

$$
\begin{aligned}
\Phi_{\lambda}(u, v)= & \frac{1}{2} \int_{\mathbb{R}^{N}}|\xi|^{2 s}|\mathcal{F} u(\xi)|^{2} d \xi+\frac{1}{2} \int_{\mathbb{R}^{N}} V(x) u^{2} d x+\frac{1}{2} \int_{\mathbb{R}^{N}}|\xi|^{2 s}|\mathcal{F} v(\xi)|^{2} d \xi \\
& +\frac{1}{2} \int_{\mathbb{R}^{N}} V(x) v^{2} d x-\lambda \int_{\mathbb{R}^{N}} F(x, u, v) d x \\
= & \frac{1}{2}\|(u, v)\|^{2}-\lambda \int_{\mathbb{R}^{N}} F(x, u, v) d x \\
\leq & \frac{1}{2}\|(u, v)\|^{2}-\lambda \int_{D_{(u, v)}} F(x, u, v) d x \\
\leq & \frac{1}{2}\|(u, v)\|^{2}-\lambda \int_{D_{(u, v)}} \frac{1}{\varepsilon_{k}^{3}}|(u, v)|^{2} d x \\
\leq & \frac{1}{2}\|(u, v)\|^{2}-\lambda \varepsilon_{k}^{2}\|(u, v)\|^{2} \frac{\operatorname{meas}\left(D_{(u, v)}\right)}{\varepsilon_{k}^{3}} \\
\leq & \frac{1}{2}\|(u, v)\|^{2}-\lambda\|(u, v)\|^{2} .
\end{aligned}
$$

Because $\lambda \in[1,2]$,

$$
\Phi_{\lambda}(u, v) \leq \frac{1}{2}\|(u, v)\|^{2}-\|(u, v)\|^{2}=-\frac{1}{2}\|(u, v)\|^{2}
$$

Now, we only need to choose $\rho_{k}>\max \left\{r_{k}, \frac{R_{k}}{\varepsilon_{k}}\right\}$; one has

$$
a_{k}(\lambda)=\max _{(u, v) \in \mathcal{Y}_{k},\|(u, v)\|=\rho_{k}} \Phi_{\lambda}(u, v)=-\frac{\rho_{k}}{2}<0,
$$

where $k \in N$ and $\lambda \in[1,2]$. The proof is completed.

Theorem 3.4 Assume $\left(f_{1}\right)-\left(f_{5}\right)$ hold, $F_{u}(x,-u,-v)=-F_{u}(x, u, v)$ and $F_{v}(x,-u,-v)=$ $-F_{v}(x, u, v)$ for $(x, u, v) \in \mathbb{R}^{N} \times H \times H$. Then the system (1.1) possesses infinitely many high energy solutions $\left(u^{k}, v^{k}\right) \in H \times H$ for all $k \geq K_{0}$ with $K_{0} \in N$, i.e., as $k \rightarrow+\infty$

$$
\begin{aligned}
\Phi_{\lambda}\left(u^{k}, v^{k}\right)= & \frac{1}{2} \int_{\mathbb{R}^{N}}|\xi|^{2 s}\left|\mathcal{F} u^{k}(\xi)\right|^{2} d \xi+\frac{1}{2} \int_{\mathbb{R}^{N}} V(x)\left|u^{k}\right|^{2} d x+\frac{1}{2} \int_{\mathbb{R}^{N}}|\xi|^{2 s}\left|\mathcal{F} v^{k}(\xi)\right|^{2} d \xi \\
& +\frac{1}{2} \int_{\mathbb{R}^{N}} V(x)\left|v^{k}\right|^{2} d x-\lambda \int_{\mathbb{R}^{N}} F\left(x, u^{k}, v^{k}\right) d x \\
\rightarrow & +\infty
\end{aligned}
$$

Proof By the hypothesis $\left(f_{1}\right)$, we conclude that $B(u, v) \geq 0$ for all $(u, v) \in H \times H$ and $A(u, v) \rightarrow+\infty$ as $\|(u, v)\| \rightarrow+\infty$. Furthermore, $\Phi_{\lambda}(-u,-v)=\Phi_{\lambda}(u, v)$ for $(u, v) \in H \times H$ and $\lambda \in[1,2]$. Considering $\left(f_{4}\right),\left(f_{5}\right)$ and Lemma 2.1, $\Phi_{\lambda}(u, v)$ maps a bounded set into a bounded set uniformly for any $\lambda \in[1,2]$. By Lemma 3.3, we can verify $\left(A_{3}\right),\left(A_{4}\right)$ of Theorem 2.3. Consequently, from Theorem 2.3 , there exists a sequence $\left\{\left(u_{n}^{k}(\lambda), v_{n}^{k}(\lambda)\right)\right\}_{n=1}^{\infty}$ for $\lambda \in[1,2]$ such that

$$
\sup _{n}\left\|\left(u_{n}^{k}(\lambda), v_{n}^{k}(\lambda)\right)\right\|<\infty, \quad \Phi_{\lambda}^{\prime}\left(u_{n}^{k}(\lambda), v_{n}^{k}(\lambda)\right) \rightarrow 0
$$


and

$$
\Phi_{\lambda}\left(u_{n}^{k}(\lambda), v_{n}^{k}(\lambda)\right) \rightarrow c_{k}(\lambda)
$$

as $n \rightarrow \infty$. By Theorem 2.2 and (3.11), for $p, q>2$, we see

$$
c_{k}(\lambda) \geq b_{k}(\lambda) \geq \frac{1}{8} r_{k}^{2}=\bar{b}_{k} \rightarrow+\infty \quad \text { as } k \rightarrow+\infty .
$$

In addition

$$
c_{k}(\lambda)=\inf _{\gamma \in \Gamma_{k}} \max _{(u, v) \in B_{k}} \Phi_{\lambda}(\gamma(u, v)) \leq \max _{(u, v) \in B_{k}} \Phi_{1}(u, v)=\bar{c}_{k} .
$$

Therefore

$$
\bar{b}_{k} \leq c_{k}(\lambda) \leq \bar{c}_{k}
$$

where $k>K_{0}$. By (3.18) and (3.19), we can choose a sequence $\lambda_{m} \rightarrow 1$, as $m \rightarrow \infty$, then the sequence $\left\{\left(u_{n}^{k}\left(\lambda_{m}\right), v_{n}^{k}\left(\lambda_{m}\right)\right)\right\}_{n=1}^{\infty}$ is bounded. Obviously, the sequence $\left\{\left(u_{n}^{k}\left(\lambda_{m}\right), v_{n}^{k}\left(\lambda_{m}\right)\right)\right\}_{n=1}^{\infty}$ has a strong convergent subsequence as $n \rightarrow \infty$. Hence, for $m \in N$ and $k>K_{0}$, we suppose $u_{n}^{k}\left(\lambda_{m}\right) \rightarrow u^{k}\left(\lambda_{m}\right), v_{n}^{k}\left(\lambda_{m}\right) \rightarrow v^{k}\left(\lambda_{m}\right)$ as $n \rightarrow+\infty$. By (3.18)-(3.21), one has

$$
\left\langle\Phi_{\lambda_{m}}^{\prime}\left(u^{k}\left(\lambda_{m}\right), v^{k}\left(\lambda_{m}\right)\right),\left(u^{k}\left(\lambda_{m}\right), v^{k}\left(\lambda_{m}\right)\right)\right\rangle=0
$$

and

$$
\Phi_{\lambda_{m}}\left(u^{k}\left(\lambda_{m}\right), v^{k}\left(\lambda_{m}\right)\right) \in\left[\bar{b}_{k}, \bar{c}_{k}\right]
$$

for $k>K_{0}$. By Lemma 3.5, $\left\{\left(u^{k}\left(\lambda_{m}\right), v^{k}\left(\lambda_{m}\right)\right)\right\}_{m=1}^{\infty}$ has a strong convergent subsequence with $u^{k}\left(\lambda_{m}\right) \rightarrow u^{k}, v^{k}\left(\lambda_{m}\right) \rightarrow v^{k}$ for $k>k_{0}$. Consequently, the $\left(u^{k}, v^{k}\right)$ is the critical point of $\Phi\left(u^{k}, v^{k}\right)=\Phi_{1}\left(u^{k}, v^{k}\right)$ with $\Phi\left(u^{k}, v^{k}\right) \in\left[\bar{b}_{k}, \bar{c}_{k}\right]$. Since $\bar{b}_{k} \rightarrow+\infty$ as $k \rightarrow+\infty$, we get infinitely many nontrivial solutions with high energy for systems (1.1). The proof is completed.

Lemma $3.5\left\{\left(u_{n}^{k}\left(\lambda_{m}\right), v_{n}^{k}\left(\lambda_{m}\right)\right)\right\}_{n=1}^{\infty}$ is bounded in $H \times H$.

Proof We argue by contradiction. Suppose that $\left\|\left(u_{n}^{k}\left(\lambda_{m}\right), v_{n}^{k}\left(\lambda_{m}\right)\right)\right\| \rightarrow \infty$ as $n \rightarrow \infty$. We consider $\left(\tau_{n}, \omega_{n}\right):=\frac{\left(u_{n}^{k}\left(\lambda_{m}\right), v_{n}^{k}\left(\lambda_{m}\right)\right)}{\left\|\left(u_{n}^{k}\left(\lambda_{m}\right), v_{n}^{k}\left(\lambda_{m}\right)\right)\right\|}$. Then, up to a subsequence, we get

$$
\begin{aligned}
& \tau_{n} \rightarrow \tau, \quad \omega_{n} \rightarrow \omega \quad \text { in } H \times H, \\
& \tau_{n} \rightarrow \tau, \quad \omega_{n} \rightarrow \omega \quad \text { in } L^{t}\left(\mathbb{R}^{N}\right) \times L^{t}\left(\mathbb{R}^{N}\right), \\
& \tau_{n}(x) \rightarrow \tau(x), \quad \omega_{n}(x) \rightarrow \omega(x) \quad \text { a.e. } x \in \mathbb{R}^{N} .
\end{aligned}
$$

We consider two cases: 
Case 1: If $|(\tau, \omega)| \neq 0$ in $H \times H$. Since $\left\langle\Phi_{\lambda_{m}}^{\prime}\left(u_{n}^{k}\left(\lambda_{m}\right), v_{n}^{k}\left(\lambda_{m}\right)\right),\left(u_{n}^{k}\left(\lambda_{m}\right), v_{n}^{k}\left(\lambda_{m}\right)\right)\right\rangle=0$, one has

$$
\begin{aligned}
0= & \left\langle\Phi_{\lambda_{m}}^{\prime}\left(u_{n}^{k}\left(\lambda_{m}\right), v_{n}^{k}\left(\lambda_{m}\right)\right),\left(u_{n}^{k}\left(\lambda_{m}\right), v_{n}^{k}\left(\lambda_{m}\right)\right)\right\rangle \\
= & \left\|\left(u_{n}^{k}\left(\lambda_{m}\right), v_{n}^{k}\left(\lambda_{m}\right)\right)\right\|^{2} \\
& -\lambda_{m} \int_{\mathbb{R}^{N}}\left[F _ { u } \left(x, u_{n}^{k}\left(\lambda_{m}\right), v_{n}^{k}\left(\lambda_{m}\right) u_{n}^{k}\left(\lambda_{m}\right)+F_{v}\left(x, u_{n}^{k}\left(\lambda_{m}\right), v_{n}^{k}\left(\lambda_{m}\right) v_{n}^{k}\left(\lambda_{m}\right)\right] d x .\right.\right.
\end{aligned}
$$

Thus, by Fatou's lemma and conditions $\left(f_{3}\right)$ and $\left(f_{4}\right)$

$$
\begin{aligned}
1= & \lambda_{m} \int_{\mathbb{R}^{N}} \frac{1}{\left\|\left(u_{n}^{k}\left(\lambda_{m}\right), v_{n}^{k}\left(\lambda_{m}\right)\right)\right\|^{2}}\left[F _ { u } \left(x, u_{n}^{k}\left(\lambda_{m}\right), v_{n}^{k}\left(\lambda_{m}\right) u_{n}^{k}\left(\lambda_{m}\right)\right.\right. \\
& +F_{v}\left(x, u_{n}^{k}\left(\lambda_{m}\right), v_{n}^{k}\left(\lambda_{m}\right) v_{n}^{k}\left(\lambda_{m}\right)\right] d x \\
\geq & \lambda_{m} \mu \int_{\mathbb{R}^{N}} \frac{F\left(x, u_{n}^{k}\left(\lambda_{m}\right), v_{n}^{k}\left(\lambda_{m}\right)\right)}{\left\|\left(u_{n}^{k}\left(\lambda_{m}\right), v_{n}^{k}\left(\lambda_{m}\right)\right)\right\|^{2}} d x \\
= & \lambda_{m} \mu \int_{\mathbb{R}^{N}}\left|\left(\tau_{n}, \omega_{n}\right)\right|^{2} \frac{F\left(x, u_{n}^{k}\left(\lambda_{m}\right), v_{n}^{k}\left(\lambda_{m}\right)\right)}{\left|\left(u_{n}^{k}\left(\lambda_{m}\right), v_{n}^{k}\left(\lambda_{m}\right)\right)\right|^{2}} d x \rightarrow+\infty \text { as } n \rightarrow+\infty .
\end{aligned}
$$

This is a contradiction.

Case 2: If $|(\tau, \omega)|=0$ in $H \times H$. By (3.22), (3.23) and $\left(f_{4}\right)$, we obtain

$$
\begin{aligned}
& \mu \Phi_{\lambda_{m}}\left(u_{n}^{k}\left(\lambda_{m}\right), v_{n}^{k}\left(\lambda_{m}\right)\right)-\left\langle\Phi_{\lambda_{m}}^{\prime}\left(u_{n}^{k}\left(\lambda_{m}\right), v_{n}^{k}\left(\lambda_{m}\right)\right),\left(u_{n}^{k}\left(\lambda_{m}\right), v_{n}^{k}\left(\lambda_{m}\right)\right)\right\rangle \\
&=\left(\frac{\mu}{2}-1\right)\left\|\left(u_{n}^{k}\left(\lambda_{m}\right), v_{n}^{k}\left(\lambda_{m}\right)\right)\right\|^{2} \\
& \quad+\lambda_{m} \int_{\mathbb{R}^{N}}\left[F _ { u } \left(x, u_{n}^{k}\left(\lambda_{m}\right), v_{n}^{k}\left(\lambda_{m}\right) u_{n}^{k}\left(\lambda_{m}\right)+F_{v}\left(x, u_{n}^{k}\left(\lambda_{m}\right), v_{n}^{k}\left(\lambda_{m}\right) v_{n}^{k}\left(\lambda_{m}\right)\right.\right.\right. \\
& \quad-\mu F\left(x, u_{n}^{k}\left(\lambda_{m}\right), v_{n}^{k}\left(\lambda_{m}\right)\right] d x . \\
& \geq\left(\frac{\mu}{2}-1\right)\left\|\left(u_{n}^{k}\left(\lambda_{m}\right), v_{n}^{k}\left(\lambda_{m}\right)\right)\right\|^{2}+\lambda_{m} \int_{\mathbb{R}^{N}} c\left(1+\left|\left(u_{n}^{k}\left(\lambda_{m}\right), v_{n}^{k}\left(\lambda_{m}\right)\right)\right|^{2}\right) d x .
\end{aligned}
$$

Therefore,

$$
\begin{aligned}
& \frac{\mu \Phi_{\lambda_{m}}\left(u_{n}^{k}\left(\lambda_{m}\right), v_{n}^{k}\left(\lambda_{m}\right)\right)-\left\langle\Phi_{\lambda_{m}}^{\prime}\left(u_{n}^{k}\left(\lambda_{m}\right), v_{n}^{k}\left(\lambda_{m}\right)\right),\left(u_{n}^{k}\left(\lambda_{m}\right), v_{n}^{k}\left(\lambda_{m}\right)\right)\right\rangle}{\left\|\left(u_{n}^{k}\left(\lambda_{m}\right), v_{n}^{k}\left(\lambda_{m}\right)\right)\right\|^{2}} \\
& \quad \geq\left(\frac{\mu}{2}-1\right)+\lambda_{m} \int_{\mathbb{R}^{N}} c\left(\frac{1+\left|\left(u_{n}^{k}\left(\lambda_{m}\right), v_{n}^{k}\left(\lambda_{m}\right)\right)\right|^{2}}{\left\|\left(u_{n}^{k}\left(\lambda_{m}\right), v_{n}^{k}\left(\lambda_{m}\right)\right)\right\|^{2}}\right) d x . \\
& \quad=\left(\frac{\mu}{2}-1\right)+\lambda_{m} \int_{\mathbb{R}^{N}} c\left(\frac{1}{\left\|\left(u_{n}^{k}\left(\lambda_{m}\right), v_{n}^{k}\left(\lambda_{m}\right)\right)\right\|^{2}}+\frac{\left|\left(u_{n}^{k}\left(\lambda_{m}\right), v_{n}^{k}\left(\lambda_{m}\right)\right)\right|^{2}}{\left\|\left(u_{n}^{k}\left(\lambda_{m}\right), v_{n}^{k}\left(\lambda_{m}\right)\right)\right\|^{2}}\right) d x \\
& =\left(\frac{\mu}{2}-1\right)+\lambda_{m} \int_{\mathbb{R}^{N}} c\left(\frac{1}{\left\|\left(u_{n}^{k}\left(\lambda_{m}\right), v_{n}^{k}\left(\lambda_{m}\right)\right)\right\|^{2}}+\left|\left(\tau_{n}, \omega_{n}\right)\right|^{2}\right) d x .
\end{aligned}
$$

Letting $n \rightarrow+\infty$, we get $0 \geq \frac{\mu}{2}-1$, i.e. $\mu \leq 2$; this is a contradiction with the hypothesis $\mu>2$. Therefore, $\left\{\left(u_{n}^{k}\left(\lambda_{m}\right), v_{n}^{k}\left(\lambda_{m}\right)\right)\right\}_{n=1}^{\infty}$ is bounded in $H \times H$. 


\section{Acknowledgements}

The authors express their sincere gratitude to the editors and anonymous referees for their constructive comments and suggestions that helped to improve the presentation of the results and accentuate important details. The paper is supported by National natural Science Foundation of China (No. 11571197).

\section{Funding}

The work is supported by National Natural Science Foundation of China (No. 11571197).

\section{Availability of data and materials}

Not applicable.

\section{Ethics approval and consent to participate}

Not applicable.

\section{Competing interests}

The authors declare that they have no competing interests.

\section{Consent for publication}

Not applicable.

\section{Authors' contributions}

All authors read and approved the final manuscript.

\section{Author details}

'School of Mathematical Sciences, Qufu Normal University, Qufu, China. ${ }^{2}$ Qingdao University of Technology, Feixian, China.

\section{Publisher's Note}

Springer Nature remains neutral with regard to jurisdictional claims in published maps and institutional affiliations.

Received: 16 August 2019 Accepted: 11 June 2020 Published online: 19 June 2020

\section{References}

1. Salahshour, S., Ahmadian, A., Salimi, M., Ferrara, M., Baleanu, D.: Asymptotic solutions of fractional interval differential equations with nonsingular kernel derivative. Chaos, Interdiscip. J. Nonlinear Sci. 29(8), Article ID 083110 (2019)

2. Ahmadian, A., Salahshour, S., Chan, C.S.: Fractional differential systems: a fuzzy solution based on operational matrix of shifted Chebyshev polynomials and its applications. IEEE Trans. Fuzzy Syst. 25(1), 218-236 (2017)

3. Agarwal, P., Dragomir, S.S., Jleli, M., Samet, B.: Advances in Mathematical Inequalities and Applications. Birkhäuser, Basel (2018)

4. Ruzhansky, M., Cho, Y.J., Agarwal, P., Area, I.: Advances in Real and Complex Analysis with Applications. Springer, Singapore (2017)

5. Agarwal, P., Baleanu, D., Chen, Y., Momani, S., Machado, J.A.T. (eds.): Fractional Calculus: ICFDA 2018, Amman, Jordan, July 16-18. Springer Proceedings in Mathematics and Statistics, vol. 303 (2019)

6. Teng, K.M.: Multiple solutions for a class of fractional Schrödinger equations in $\mathbb{R}^{N}$. Nonlinear Anal., Real World Appl. 21, 79-86 (2015)

7. Du, X.S., Mao, A.M.: Existence and multiple of nontrivial solutions for a class of fractional Schrödinger equations. J. Funct. Spaces 2017, Article ID 3793872 (2017)

8. Chang, S.Y.A., González, M.M.: Fractional Laplacian in conformal geometry. Adv. Math. 226, 1410-1432 (2011)

9. Caffarelli, L., Silvestre, L.: An extension problem related to the fractional Laplacian. Commun. Partial Differ. Equ. 32 1245-1260 (2007)

10. Chang, X., Wang, Z.Q.: Ground state of scalar field equations involving a fractional Laplacian with general nonlinearity Nonlinearity 26, 479-494 (2013)

11. Mao, A.M., Yang, L., Qian, A.X., Luan, S.X.: Existence and concentration of solutions of Schrödinger-Poisson system. Appl. Math. Lett. 68, 8-12 (2017)

12. Dipierro, S., Palatucci, G., Valdinoci, E.: Existence and symmetry results for a Schrödinger type problem involving the fractional Laplacian. Matematiche LXVIII, 201-216 (2013)

13. Laskin, N.: Fractional quantum mechanics and Lévy path integrals. Phys. Lett. A 268, 298-305 (2000)

14. Laskin, N.: Fractional Schrödinger equation. Phys. Rev. 66, 56-108 (2002)

15. Liu, Z.S., Guo, S.J.: On ground state solutions for the Schrödinger-Poisson equations with critical growth. J. Math. Anal. Appl. 412, 435-448 (2014)

16. Di Nezza, E., Palatucci, G., Valdinoci, E.: Hitchhiker's guide to the fractional Sobolev spaces. Bull. Sci. Math. 136 521-573 (2012)

17. Silvestre, L.: Regularity of the obstacle problem for a fractional power of the Laplace operator. Commun. Pure Appl. Math. 60, 67-112 (2007)

18. Secchi, S.: Ground state solutions for nonlinear fractional Schrödinger equations in $\mathbb{R}^{N}$. J. Math. Phys. 54, Article ID 031501 (2013)

19. Felmer, P., Quaas, A., Tan, J.: Positive solutions of nonlinear Schrödinger equation with the fractional Laplacian. Proc. R Soc. Edinb., Sect. A 142, 1237-1262 (2012)

20. Shang, X.D., Zhang, J.H.: Ground states for fractional Schrödinger equations with critical growth. Nonlinearity 27 187-207 (2014) 
21. Servadei, R., Valdinoci, E.: The Brezis-Nirenberg result for the fractional Laplacian. Trans. Am. Math. Soc. 367, 67-102 (2015)

22. Teng, K.M., He, X.M.: Ground state solutions for fractional Schrödinger equations with critical Sobolev exponent Commun. Pure Appl. Anal. 15, 991-1008 (2016)

23. Zhao, L.G., Zhao, F.K.: On the existence of solutions for the Schrödinger-Poisson equations. J. Math. Anal. Appl. 346, 155-169 (2008)

24. Berestycki, H., Lions, P.L.: Nonlinear scalar field equations. Arch. Ration. Mech. Anal. 82, 313-379 (1983)

25. Rabinowitz, P.H.: On a class of nonlinear Schrödinger equations. Z. Angew. Math. Phys. 43, 270-291 (1992)

26. Strauss, W.A.: Existence of solitary waves in higher dimensions. Commun. Math. Phys. 55, 149-162 (1977)

27. Bartsch, T., Wang, Z., Willem, M.: The Dirichlet problem for superlinear elliptic equations. In: Handbook of Differentia Equations-Stationary Partial Differential Equations, vol. 2, pp. 1-55 (2005)

28. Zhang, J.H., Zhang, Z.T.: Existence results for some nonlinear elliptic systems. Nonlinear Anal. 71, 2840-2846 (2009)

29. Cao, D.M., Tang, Z.W.: Solutions with prescribed number of nodes to superlinear elliptic systems. Nonlinear Anal. 55 , 707-722 (2003)

30. Pomponio, A.: Asymptotically linear cooperative elliptic system: existence and multiplicity. Nonlinear Anal. 52 989-1003 (2003)

31. Zhao, P.H., Wang, X.Y.: The existence of positive solution of elliptic system by a linking theorem on product space. Nonlinear Anal. 56, 227-240 (2004)

32. Boccardo, L., de Figueiredo, D.G.: Some remarks on a system of quasilinear elliptic equations. Nonlinear Differ. Equ. Appl. 9, 309-323 (2002)

33. Alves, C.O., de Morais Filho, D.C., Miyagaki, O.H.: Multiple solutions for an elliptic system on bounded and unbounded domains. Nonlinear Anal. 56, 555-568 (2004)

34. Zou, H.H.: A priori estimates for a semilinear elliptic system without variational structure and their applications. Math. Ann. 323, 713-735 (2002)

35. Chang, K.C.: Principal eigenvalue for weight matrix in elliptic systems. Nonlinear Anal. 46, 419-433 (2001)

36. Zhang, Z.T., Wang, W.: Structure of positive solutions to a Schrödinger system. J. Fixed Point Theory Appl. 19, 877-887 (2016)

37. Liu, H.D., Liu, Z.L.: Multiple positive solutions of elliptic systems in exterior domains. Commun. Contemp. Math. 2017 Article ID 1750063 (2017)

38. Qi, Z.X., Zhang, Z.T.: Existence of multiple solutions to a class of nonlinear Schrödinger system with external sources terms. J. Math. Anal. Appl. 420, 972-986 (2014)

39. Zou, W.: Variant fountain theorems and their applications. Manuscr. Math. 104, 343-358 (2001)

40. Willem, M.: Minimax Theorems. Birkhäuser, Boston (1996)

\section{Submit your manuscript to a SpringerOpen ${ }^{\circ}$ journal and benefit from:}

- Convenient online submission

- Rigorous peer review

- Open access: articles freely available online

- High visibility within the field

- Retaining the copyright to your article

Submit your next manuscript at $\boldsymbol{\nabla}$ springeropen.com 